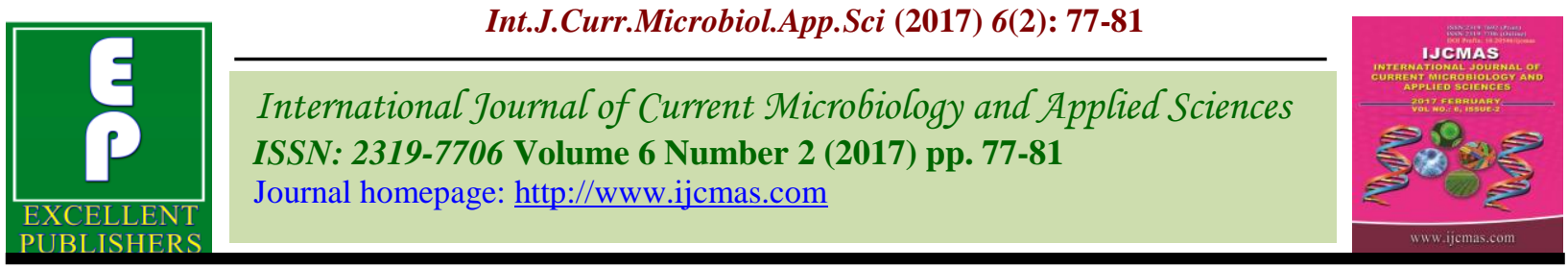

Original Research Article

http://dx.doi.org/10.20546/ijcmas.2017.602.010

\title{
Rapid Detection of Mycobacteria using automated Detection/ Incubation System in Exudative Pleural Effusion in Alexandria Main University Hospital
}

\author{
Ahmed Youssef Shaaban ${ }^{1}$ and Nesrine Fathi Hanafi ${ }^{2 *}$ \\ ${ }^{1}$ Chest Department, Faculty of Medicine, Alexandria University, Egypt \\ ${ }^{2}$ Medical Microbiology and Immunology, Faculty of Medicine, Alexandria University, Egypt \\ *Corresponding author
}

A B S T R A C T

\begin{abstract}
Keywords
Tuberculosis,

Tuberculous

pleural effusion

fluid,

Mycobacterial

bacilli.

Article Info

Accepted:

05 January 2017

Available Online:

10 February 2017

Studies have been targeting to evaluate newer automated culture systems techniques for different types of samples to provide a more rapid diagnosis relying on sensitive and specific principle with the ability to discriminate between viable and dead mycobacterial bacilli. This study aimed at assessment of bactalert, biomerieux $\mathbb{B}$ culture system as a rapid diagnostic tool for tuberculous pleural effusion fluid in comparison with Lowenstein Jensen conventional culture and Zeil Nelseen stained smears. Pleural aspirate collected from 60 patients presented with exudative pleural effusion was centrifuges and the deposit divided to be inoculated on bactalert bottles, on Lowenstein Jensen media and the third part was fixed on a slide and stained with Zeil Nelseen stain. Our study confirmed previous reports that bact Alert system has very high sensitivity still with lower specificity. This finding might consider Bact Alert system a reliable, easy, rapid screening test for tuberculous pleurisy that needs confirmation via other laboratory assays.
\end{abstract}

\section{Introduction}

Tuberculosis has been long considered as the leading curable infectious disease causing death (Dye et al., 2008; Kumar, 2015). Studies in populations with a high prevalence of tuberculosis reported that $30 \%$ of patients with tuberculosis are associated with tuberculous pleurisy as one of the most common forms of extrapulmonary tuberculosis (TB) (Aljohaney et al., 2012; WHO, 2013). The pathogenesis of primary TB effusion was described to be mainly due to delayed hypersensitivity response to mycobacterial antigens and activation of pleural cells for cytokine production. Many studies have investigated the usefulness of measuring different parameters in pleural fluid for an early diagnosis of tuberculous pleurisy. The definitive diagnosis of TB pleural effusions depends on the demonstration of acid-fast bacilli in the sputum, pleural fluid, or pleural biopsy specimens (Lima et al., 2003). The diagnosis can be established in a majority of patients from the clinical features, pleural fluid examination, including cytology, biochemistry, and bacteriology, and pleural biopsy. Culture on Lowenstein Jensen media is considered as the gold standard for definite diagnosis still it needs as long as 42 days to reveal a result. Detection of Acid fast bacilli via Zeil Neelsen staining of smears of pleural 
fluid have been considered to be a rapid diagnostic tool but with a low sensitivity as well as inability to discriminate between actively replicating and dead bacilli (Steingart et al., 2007; Uddin et al., 2013). Similarly, rapid molecular techniques as PCR for detection of TB nucleic acid lacked the ability to discriminate between active and inactive TB cases positive for mycobacterial DNA. On the other hand, cytologic examination inspite specific had a reported low sensitivity while biochemical assays including adenosine deaminase and interferon gamma dectction in the pleural fluid lacked specificity inspite of their very high sensitivity (Kaisemann et al., 2004; Tay et al., 2013).

Studies have been targeting to evaluate newer culture systems techniques to provide a more rapid diagnosis relying on sensitive and specific principle with the ability to discriminate between viable and dead mycobacterial bacilli. We intended in this study to assess bactalert culture system as a rapid diagnostic tool for tuberculous pleural effusion in comparison with Lowenstein Jensen and Zeil Neelsen stained smears.

\section{Patients and Methods}

60 patients presented to the department of chest medicine Alexandria main University hospital diagnosed as exudative pleural effusion according to light's criteria (Light $e t$ al.,, 1973; Light, 2002), have been counseled and consented for the requirements of the study. In case of approval, Data concerning age, previous medical history, signs and symptoms have been collected from the patients.

Pleural effusion fluid has been collected from all patients via thoracocentesis under strict aseptic technique in heparinized syringe to avoid clotting. Centrifugation was performed in sterile tubes for 10 minutes at 5000Xrpm, supernatant have been discarded less the lowermost $1 \mathrm{ml}$. The deposit has been suspended in this $1 \mathrm{ml}$ and divided into three parts:

$500 \mathrm{ml}$ was inoculated on bactalert MB enrichment (fluid culture media with supplement) vials kit (bioMérieux, USA) following the manufacturer instructions and inserted into Bactalert incubation/detection system (bioMérieux, USA) results were considered positive when a positive alert was revealed from the detection system and a growth curve was demonstrated via software (Metchock et al., 1999).

$300 \mathrm{ml}$ were used to make a direct smear subjected to Zeil Neelsen staining and examination by oil immersion lens under the light microscopy results were considered positive when acid fast bacilli were revealed (Master, 1992).

200ml have been inoculated on Lowenstein Jensen media (sigma-Aldrich, USA). Results were considered positive in case of growth of colonies that were identified for confirmation to be a mycobacterium tuberculosis growth (Master, 1992; Lowenstein, 1931).

\section{Results and Discussion}

Among the patients enrolled in the study 28(46.6\%) were males and 32 (54.4\%) were females, those results agree with similar reports noting a higher female to male ration (Fader et al., 2010; Noertjojo et al., 2002; Forssbohm et al., 2008) with an age range 2580 years with the mean + SD 49.23+12.6, these results were confirming those of previous studies reporting approximately similar means of ages for patients presenting with pleural effusion (Hasaneen et al., 2003).

Highest rate of positivity among pleural effusion samples was revealed by the BACT/ 
ALERT incubation/detection system, and the lowest posititvity detection was by direct $\mathrm{ZN}$ smear, this might be justified by small number of acid fast bacilli within different samples as it depends on whether or not the caseating lesions have got access to open in the pleura cavity or not. Similarly, reports from numerous researches have revealed different sensitivities for Zeil Neelsen direct smear (Lai et al., 2012; Maurya et al., 2011; Gill et al., 2013; Uddin et al., 2013).

Considering direct Zeil Neelsen smear as gold standard for comparison, it was revealed that the sensitivity of the results of bact/alert incubation / detection system (100\%), specificity $(76.5 \%)$, Positive predictive value (42.8\%), Negative predictive value (76.4\%), Accuracy $(71 \%)$.

Considering LJ culture as gold standard for comparison, it was revealed that the sensitivity of the results of bact/alert incubation/detection system $(100 \%)$, specificity $(95.1 \%)$, positive predictive value $(90.47 \%)$, negative predicitive value $(100 \%)$, accuracy $(88 \%)$ (Table 1$)$.

Table.1 Comparison of the results of the performed laboratory assays

\begin{tabular}{|l|l|l|l|}
\hline & Bact/alert & $\begin{array}{l}\text { Lowenstein Jensen } \\
\text { media } \\
\mathrm{n}=60\end{array}$ & $\begin{array}{l}\text { Direct Zeil Neelsen } \\
\text { smear } \\
\mathrm{n}=60\end{array}$ \\
\hline Positive & $21(35 \%)$ & $19(31.67 \%)$ & $9(15 \%)$ \\
\hline negative & $39(65 \%)$ & $41(68.33 \%)$ & $51(85 \%)$ \\
\hline
\end{tabular}

Table. 2 Comparison of bact/alert results versus direct Zeil Neelsen smear

\begin{tabular}{|l|l|l|l|}
\hline \multirow{2}{*}{ Bact/alert } & \multicolumn{2}{|l|}{ Direct Zeil Neelsen smear } & \\
\cline { 2 - 4 } & Positive $(\mathrm{n}=9)$ & Negative $(\mathrm{n}=51)$ & Total $(\mathrm{n}=60)$ \\
\hline Positive $(\mathrm{n}=21)$ & $9(100 \%)$ & $12(23.5 \%)$ & $21(35 \%)$ \\
\hline Negative $(\mathrm{n}=39)$ & $0(0 \%)$ & $39(76.5 \%)$ & $39(65 \%)$ \\
\hline
\end{tabular}

Table.3 Comparison of bact/alert results versus Lowenstein Jensen culture results

\begin{tabular}{|l|l|l|l|}
\hline \multirow{2}{*}{ Bact/alert } & \multicolumn{2}{|l|}{ Lowenstein Jensen culture } & \\
\cline { 2 - 4 } & Positive $(\mathrm{n}=19)$ & Negative $(\mathrm{n}=41)$ & Total $(\mathrm{n}=60)$ \\
\hline Positive $(\mathrm{n}=21)$ & $19(100 \%)$ & $2(4.9 \%)$ & $21(35 \%)$ \\
\hline Negative $(\mathrm{n}=39)$ & $0(0 \%)$ & $39(95.1 \%)$ & $39(65 \%)$ \\
\hline
\end{tabular}

Both data in table 2 and 3 show a very high sensitivity ( $100 \%$ ) of BACT/ALERT incubation /detection system for detection of mycobacterium TB in exudative pleural effusion still with a lower specificity $(76.5 \%$ and $95.1 \%$ ) compared to Zeil-Neeleen direct smear and Lowenstein Jensen culture respectively. This agrees with data released from many researchers reporting a high false positive rate of results with many developed automated incubation detection systems (Jorgensen, 1997; Weinstein, 1996; Morgan, 1983; Ichiyama, 1993). This finding have been justified by many authors who audited automated systems results as (Alfa, 1995; Martinez and Lakshmi, 2001 and Tortoli et al., 1998) that have reported false positive signals from automated detection systems for mybobacterial growth associated with presence 
of high counts or malignant lymphocytes and polymorphs in the inoculated samples due to release of carbon dioxide from respiration of these white blood cells that might activate sensors of the detection system to reveal false positive signals.

The present study results confirm those of previous researchers that reported very high sensitivity of BACT/ART to detect mycobacterial growth with pleural effusion fluid, still its low specificity might mandate confirmation of the results using other methods with higher specificity. Due to its high sensitivity (100\%), low labour, simple methodology, and rapid results BAct/Alert might be recommended as a reliable screening method for tuberculous pleurisy.

\section{References}

Alfa, M., Sanche, S., Roman, S., Fiola, Y., Lenton, P., Harding, G. 1995. Continuous quality improvements for introduction of automated blood culture instrument. J. Clin. Microbiol., 33: 1185-91.

Aljohaney, K., Amjadi, and G.G. Alvarez. 2012. A Systematic Review of the Epidemiology, Immunopathogenesis, Diagnosis, and Treatment of Pleural TB in HIV- Infected Patients. Clin. Developmental Immunol., 19.

Dye, C., William, B.G. 2008. Eliminating human tuberculosis in the twenty-first century. $J$. R. Soc. Interface, 5: 653-62.

Fader, T., Parks, J., Khan, N.U., Manning, R., Stokes, S., Nasir, N.A. 2010. Extrapulmonary tuberculosis in Kabul Afghanistan: A hospital- based retrospective review. Int. J. Infectious Dis., 14:e 102-10.

Forssbohm, M., Zwahlen, M., Loddenkemper, R., Rieder, H. 2008. Demographic characteristics of patients with extrapulmonary tuberculosis in Germany. Eur. Respir. J., 31: 99-105.

Gill, M.K., Kukreja, S., Chabra, N. 2013. Evaluation of nested PCR for rapid diagnosis of clinically suspected tuberculosis pleurisy. J. Clin. Diag. Res.,
7(11): 2456-8.

Hasaneen, N.A., Zaki, M.E., Shalaby, H.M. and Ahmad, S. 2003. polymerase chain reaction of pleural biopsy in a rapid and sensitive method for the diagnosis of tuberculous pleural effusion. Chest, 124: 2105-11.

Ichiyama, S., Shimokata, K., Takeuchi, J. 1993. The AMR Group. Comparative study of a biphasic culture system (Roche MB check system) with a conventional egg medium for recovery of mycobacteria. Tuberc. Lung Dis., 74: 338- 41.

Jorgensen, J.H., Mirret, S., McDonald, L.C., et al. 1997. Controlled clinical laboratory comparison of BACTEC plus aerobic/resin medium with Bact/Alert aerobic FAN medium for detection of bacteremia and fungemia. J. Clin. Microbiol., 35: 53-8.

Kaisemann, M.C., Kriski, A.L., Pereira, M.F., Trajman, A. 2004. Pleural fluid adenosine deaminase detection for the diagnsosis of pleural tuberculosis. J. Bras. pneumol., 30(6): 1806-13.

Kumar, M.H. 2015. "Tuberculosis is a Fatal Disease among Some Developing Countries of the World." American J. Infectious Dis. Microbiol., 3(1): 18-31.

Lai, Y.C., Chang, S.C., Yan, M.K., Lai, J.I., Lin, P.C., Kuo, L.C., et al. 2012. Tuberculous pleural effusion in elderly. Int. $J$. Geronotol., 6: 224-8.

Lakshmi, V. 2001. Culture of body fluids using the Bact/ALERT system. Indian J. Med. Microbiol., 19(2): 67-72.

Light, R.W. 2002. Clinical practice. Pleural effusion. N. Engl. J. Med., 346(25): 1971-7.

Light, R.W., Macgregor, M.I., Luchsinger, P.C., Ball, W.C. Jr. 1972. Pleural effusions: the diagnostic separation of transudates and exudates. Ann. Intern. Med., 77(4): 507-13.

Lima, D.M., J. Keny, B. Colares, J.K. B., Da Fonseca, B.A.L. 2003. Combined Use of the Polymerase Chain Reaction and Detection of Adenosine Deaminase Activity on Pleural Fluid Improves the Rate of Diagnosis of Pleural Tuberculosis. Clinical Investigations, 124(3): 909-14.

Lowenstein, E. 1931. Die zachtung der tuberkelbazillen aus dem stramenden blute. Zentralb. Bakteriol. Parasitenkd. Infektionskr. Hyg. Abt. I Orig., 120-7. 
Martinez, R.M., Martinez, R., Partal, Y., Casas, J., Llosa, J., Almagro, M. 1993. An infrequent cause of false-positive blood cultures. Clin. Microbiol. Newslett., 15: 7-8.

Master, R.N. 1992. Clinical microbiology procedures handbook. American Society for Microbiology. J. Bacteriol., 191: 4714-21.

Maurya, A.K., Kant, S., Kushwaha, R.A.S., Nag, V.L., Kumar, M. and Shole, R.N. 2011. The advantage of using IS6110-PCR vs BACTEC culture for rapid detection of Mycobacterium tuberculosis from pleural fluid in Northern India. BioSci. Trends, 5(4): 159-64.

Metchock, B., Nolte, F.S., Wallace, Jr R.J. 1999. Mycobacterium, in Murrary PR, (ed): Manual of Clinical Microbiology, ed 7. Washington, DC, American Society for Microbiol., 399-437.

Morgan, M.A., Horstmeier, C.D., DeYoung, D.R., Robers, G.D. 1983. Comparison of a radiometric method (BACTEC) and conventional culture media for recovery of mycobacteria from smear-negative specimens. J. Clin. Microbiol., 18(2): 384.

Noertjojo, K., Tam, C., Chan, S., Chan-Yeung, M. 2002. Extrapulmonary and pulmonary tuberculosis in Hong King. Int. J. Tuberc. Lung Dis., 6: 879-86.

Steingart, K.R., Henry, M., Laal, S., Hopewell, P.C., Ramsay, A., Menzies, D., Cunningham, J., Weldingh, K., Pai, M.
2007. Commercial serological antibody detection tests for the diagnosis of pulmonary tuberculosis: a systematic review. PLOS Med., 4(6): 202.

Tay, T.R., Tee, A. 2013. Factors affecting pleural fuid adenosisne deaminase level and the implication on the diagnosis of tuberculous pleural effucsion: a retrospective cohort study. BMC Infectious Dis., 13: 546.

Tortoli, E., Cichero, M.P., Chirillo, B., Gimondo, M.R., Bono, L., Gesu, G., Simonetti, M.T., Bolpe, G., Nardi, G. and Marone, P. 1998. Multicenter comparison of ESP culture system II with BACTEC 460TB and with Lowenstein- Jensen Mediuim for Recovery of Mycobacteria from different clinical specimens including blood. J. Clin. Microbiol., 36(5) 1378-81.

Uddin, M.K.M., Chowdhury, M.R., Ahmed, S., Rahman, M.T., Khatun, R., Leth, F.V. et al. 2013. Comparison of direct versus concentrated smear microscopy in detection of pulmonary tuberculosis. BMC Research notes, 6: 291.

Weinstein, M.P. 1996. Current blood culture methods and systems: clinical concepts, technology, and interpretation of results. Clin. Infect. Dis., 23: 40-6.

World Health Organization. 2013. Geneva, Switzerland, Global tuberculosis report, http://apps.who.int/iris/bitstream/10665/913 55/1/9789241564656_eng.pdf.

\section{How to cite this article:}

Ahmed Youssef Shaaban and Nesrine Fathi Hanafi. 2017. Rapid Detection of Mycobacteria using automated Detection/ Incubation System in Exudative Pleural Effusion in Alexandria Main University Hospital. Int.J.Curr.Microbiol.App.Sci. 6(2): 77-81. doi: http://dx.doi.org/10.20546/ijcmas.2017.602.010 